\title{
High-resolution ex vivo magnetic resonance angiography: a feasibility study on biological and medical tissues
}

\author{
Anne S Rasmussen ${ }^{1}$, Henrik Lauridsen ${ }^{1,2}$, Christoffer Laustsen ${ }^{1}$, Bjarke G Jensen², Steen F Pedersen ${ }^{1}$, \\ Lars Uhrenholt ${ }^{3}$, Lene WT Boel ${ }^{3}$, Niels Uldbjerg ${ }^{4}$, Tobias Wang ${ }^{2}$, Michael Pedersen ${ }^{1 *}$
}

\begin{abstract}
Background: In biomedical sciences, ex vivo angiography is a practical mean to elucidate vascular structures three-dimensionally with simultaneous estimation of intravascular volume. The objectives of this study were to develop a magnetic resonance (MR) method for ex vivo angiography and to compare the findings with computed tomography $(\mathrm{CT})$. To demonstrate the usefulness of this method, examples are provided from four different tissues and species: the human placenta, a rice field eel, a porcine heart and a turtle.

Results: The optimal solution for ex vivo MR angiography (MRA) was a compound containing gelatine $(0.05 \mathrm{~g} / \mathrm{mL})$, the $C T$ contrast agent barium sulphate $(0.43 \mathrm{~mol} / \mathrm{L})$ and the $\mathrm{MR}$ contrast agent gadoteric acid $(2.5 \mathrm{mmol} / \mathrm{L})$. It was possible to perform angiography on all specimens. We found that ex vivo MRA could only be performed on fresh tissue because formalin fixation makes the blood vessels permeable to the MR contrast agent.

Conclusions: Ex vivo MRA provides high-resolution images of fresh tissue and delineates fine structures that we were unable to visualise by $\mathrm{CT}$. We found that MRA provided detailed information similar to or better than conventional CTA in its ability to visualize vessel configuration while avoiding interfering signals from adjacent bones. Interestingly, we found that vascular tissue becomes leaky when formalin-fixed, leading to increased permeability and extravascular leakage of MR contrast agent.
\end{abstract}

\section{Background}

In vivo angiography is frequently employed to produce a structural overview of the intravascular configuration in living tissues [1] whereas angiography of excised organs or post-mortem angiography is rarely performed [2-4]. For example, ex vivo angiography has been used successfully to identify structural components of carotid atherosclerotic plaques [5] and to visualize the renal microvasculature [6]. In addition, post mortem angiography provides information secondary to conventional autopsy in humans and animals $[7,8]$. Computed tomography angiography (CTA) can be employed with submillimetre resolution of vessels in conjunction with an intravascular contrast agent [9]. However, accurate delineation of the entire vessel configuration acquired by CTA is often complicated by the inherent absorption of

\footnotetext{
* Correspondence: michael@mr.au.dk

1 MR-research Centre, Aarhus University Hospital, Aarhus, Denmark
}

$\mathrm{X}$-rays in bones and cartilage, characterized as hyperintense areas on CTA images. Magnetic resonance angiography (MRA) is another method, where an intravascular confined paramagnetic compound generates a change in the local magnetization of water, resulting in a hyperintense signal of the streaming blood. MRA has potential advantages over CTA in its unique ability to reveal the intravascular compartment without concomitant contributions from bone and cartilage. On the other hand, the small molecules of most available magnetic resonance contrast agents leak rapidly into the interstitial compartment, hampering detailed and prolonged intravascular measurements using this technique. In clinical situations, this extravasation from the intravascular compartment is overcome by performing an MRA procedure in which images are acquired during the first pass of the agent through the arteries. However, as rapid circulation of blood in smaller species and the lack of circulation in excised organs and dead subjects 
preclude the use of this approach, there are today no available MRA techniques allowing three-dimensional (3D) high-resolution images of blood vessels in these situations.

This methodological study presents a novel method for ex vivo MRA. To demonstrate the usefulness of this method, examples of both zoological and biomedical relevance were studied from four distinctly different tissues: the human placenta, a rice field eel, a porcine heart and a turtle.

\section{Results and Discussion}

The experimental study was conducted in several steps. Prior to MRA and CTA of selected specimens, we defined the optimal concentration of contrast agents. Next, the use of formalin-fixed and fresh specimens was elucidated to reveal the microstructural consequences of protein binding in the presence of formalin. Three different specimens were then subjected to the chosen MRA and CTA protocols. First, fresh placentas were harvested following elective caesarean section from our obstetric department. Second, live specimens of rice field eel (Monopterus albus) were purchased from aquacultures in Vietnam. They were kept in a $500 \mathrm{~L}$ plastic aquarium in a $12: 12 \mathrm{~h} \mathrm{~L}: \mathrm{D}$ routine, kept at $29^{\circ} \mathrm{C}$ and fed with mussels and shrimps. Third, excised hearts were used from healthy female Danish Landrace pigs weighing $65 \mathrm{~kg}$. Fourth, turtles (Trachemys scripta) were examined to demonstrate the proposed MRA and CTA methods on a complete animal.

\section{Preparation of contrast agent}

1. An MRA protocol was applied to image tubes with various gadolinium concentrations $(0-3.5 \mathrm{mmol} / \mathrm{L})$, containing the magnetic resonance contrast agent gadoteric acid (Dotarem; Guerbet, France), to determine the concentration that generated the highest image signal. The relationship between concentration and signal is shown in Figure 1 and a concentration of $2.5 \mathrm{mmol} / \mathrm{L}$ was considered optimal with the MRA sequence and parameters given in the "Methods" section. Optimization of CT contrast concentration was not performed since development of a contrast solution for MRA was the main purpose of the study.

2. Two different contrast agent solutions were prepared. For contrast mix 1, saline was heated to $60^{\circ} \mathrm{C}$ and gelatine at a final concentration of $0.05 \mathrm{~g} / \mathrm{mL}$ (gelatine for microbiology, Merck, Darmstadt, Germany) was added

\section{Signal intensity as a function of contrast concentration}

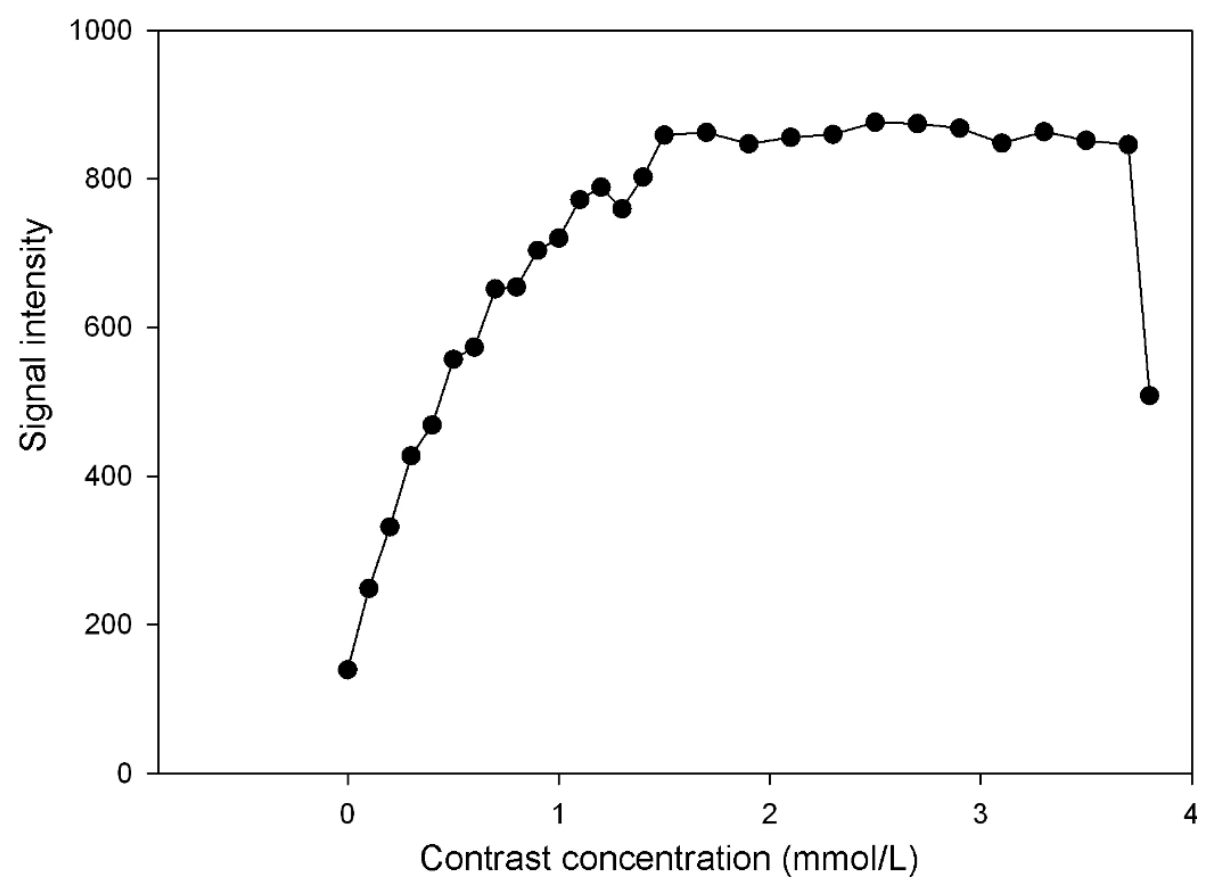

Figure 1 Optimization of concentration of the contrast agent. MR scanning of tubes containing contrast agent at different concentrations was performed. The signal intensity was plotted as a function of concentration. The signal intensity increased with contrast concentration up to $2 \mathrm{mmol} / \mathrm{L}$ followed by a steady decline so we decided to use $2.5 \mathrm{mmol} / \mathrm{L}$ in subsequent MRA studies. Data were acquired using a 3D gradientecho sequence with the following parameters: thickness $1 \mathrm{~mm}$; TR $23.1 \mathrm{~ms}$; TE $1.6 \mathrm{~ms}$ and excitation flip angle $30^{\circ}$. 
in small portions while stirring together with the CT contrast agent barium sulphate at a final concentration of $0.43 \mathrm{~mol} / \mathrm{L}$ (Mixobar Colon, $1 \mathrm{~g} / \mathrm{mL}$; Astra Tech, Sweden). Dotarem was added to a final concentration of $2.5 \mathrm{mmol} / \mathrm{L}$ when the solution had cooled to $40^{\circ} \mathrm{C}$. Contrast mix 2 contained saline and the following ingredients: the MR contrast agent gadofosveset trisodium (Vasovist; Bayer-Schering Pharma, Germany) at 2.5 $\mathrm{mmol} / \mathrm{L}$ and albumin at $60 \mathrm{~g} / \mathrm{L}$ (egg white albumin; Sigma-Aldrich, USA). The saline was heated to $40^{\circ} \mathrm{C}$, and albumin and Vasovist were added while stirring. As Vasovist binds to albumin, the gadolinium-containing compound cannot diffuse from the intra- to the extravascular compartment and Vasovist is therefore considered a suitable intravascular contrast agent for living specimens. Additionally, a commercially made CT contrast agent, Microfil (Flow Tech, USA), was used for CTA of the rice field eel head, which was compared with the acquired MRA [10].

Comparing results obtained with contrast mixes 1 and 2 (data not shown) demonstrated that contrast mix 1 was better by serving both as an MRA and a CTA agent. This property facilitates consecutive MRA and CTA on the same sample. However, contrast mix 2 may be of benefit thanks to its lower viscosity and easier distribution to small capillary vessels.

\section{Microstructural effect of formalin fixation}

Initial MRA of formalin-fixed placentas showed that the MR contrast agents easily diffused to the interstitium
(Figure 2). In contrast, MRA of fresh placentas showed that the contrast agent was confined to the blood vessels during the time of the experimental procedure. An increased permeability across the vessel endothelium was surprisingly found by scanning electron microscopy (SEM), where blood vessels from formalin-fixed and fresh placentas were investigated. SEM revealed large holes in the vessel walls of the formalin-fixed placentas, whereas the vessel walls of the fresh placentas were completely homogenous and dense (Figure 3), supporting the view that the reorganization of the vessel's microvasculature following protein binding to formalin leads to increased endothelial permeability. To our knowledge this observation is novel and could be an important consideration when performing angiography on formalin-fixed tissue in general.

\section{Human placenta}

A placenta was harvested immediately after elective caesarean section and placed in a water bath at $37^{\circ} \mathrm{C}$. The umbilical cord was cut off approximately $5 \mathrm{~cm}$ proximal to the insertion to the placenta. The amnion was cut off as well. The blood vessels of the umbilical cord were catheterized using three venous catheters (size 17). The venous catheters were fixed to the blood vessels using polyester sutures (2-0 Ethicon) and the umbilical cord was clamped using a suture to prevent reflux of the injected solutions. The placenta was then perfused using saline with $5000 \mathrm{IU} / \mathrm{L}$ heparin [11]. The solution was heated to $37^{\circ} \mathrm{C}$ and $500 \mathrm{~mL}$ was injected into the two
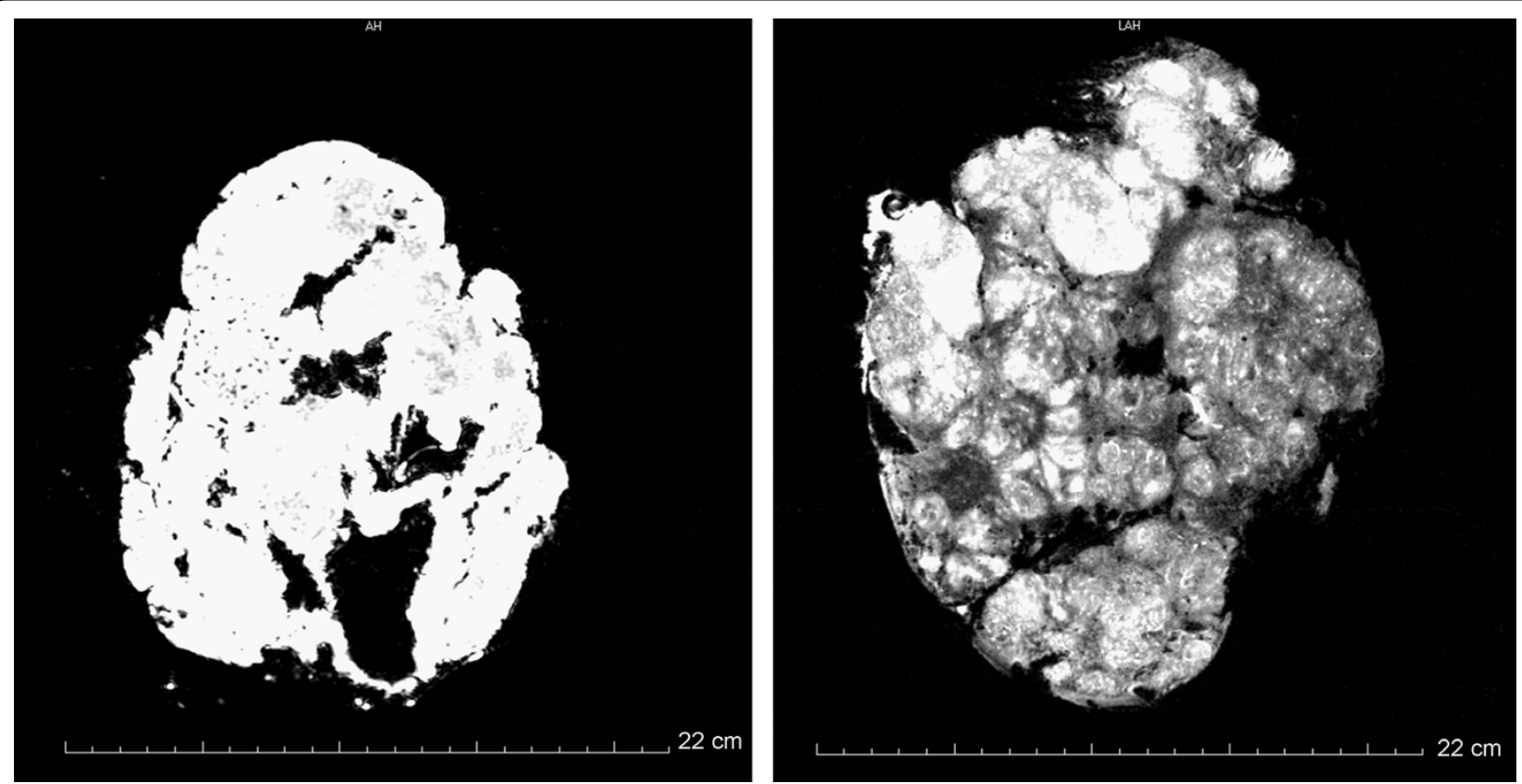

Figure 2 Left: MRA of formalin-fixed placenta prior to MRA. Right: MRA of fresh placenta. 


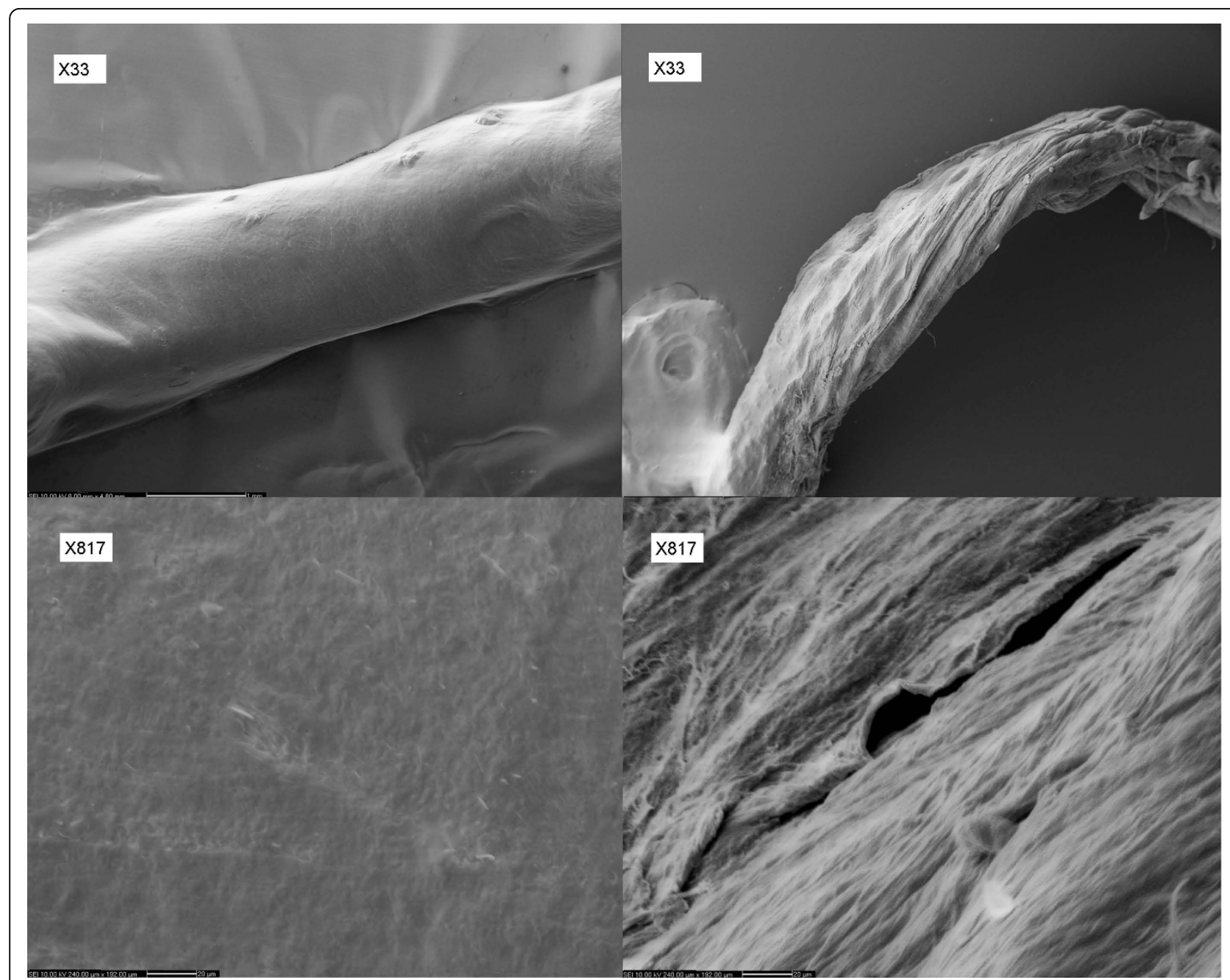

Figure 3 Left: SEM of fresh blood vessel in a placenta (top $\times \mathbf{3 3}$; bottom $\times \mathbf{8 1 7}$ ). Right: SEM of formalin-fixed blood vessels of the placenta $($ top $\times 33$; bottom $\times 817)$.

umbilical arteries with a pressure-controlled pump using the sphygmanometric principle. Contrast mix 1 was then injected using the pump with a pressure $\leq 60 \mathrm{mmHg}$ [12]. During injection, the placenta was placed in a water bath at a temperature of $35-40^{\circ} \mathrm{C}$ to ensure that the contrast solution remained liquid. It was then positioned in the MR scanner and the 3D MRA protocol was followed by subsequent fixation with $4 \%$ formalin or 2.8\% FineFix (Milestone Medical Technologies, USA). MRA showed vessels from a number of different generations starting with the cotelydonary arteries branching off into primary, secondary and tertiary stem vessels: even 6th generation blood vessels were visible. Subsequently, the placenta was subjected to CTA (Figure 4), which also showed branching of the vessels down to the 6th generation of stem vessels. In general, MRA was capable of showing considerably more very small vessels than CTA, giving the overall impression that the quality of
MRA was as good or better than the results of conventional CTA.

\section{Monopterus albus}

Specimens of Monopterus albus (weight 150-200 g; length $50-55 \mathrm{~cm}$ ) were anesthetized and euthanized with $1 \mathrm{~g} / \mathrm{L}$ benzocaine. The pericardium and ventral aorta were exposed with a ventral cut 7-12 cm caudal to the snout of the animal. The ventral aorta was catheterized and saline with $5000 \mathrm{IU} / \mathrm{L}$ heparin was infused at a pressure of $30 \mathrm{mmHg}$ [13] until all blood had been replaced. Intravascular perfusion was performed with either contrast mix 1, contrast mix 2 or Microfil. The body was cooled with ice to solidify the injected contrast agent. MRA was performed in animals injected with contrast mixes 1 or 2 (Figure 5) and CTA was performed in animals injected with Microfil (Figure 6). All MRA and CTA agents, particularly contrast mix 2 , were able to 


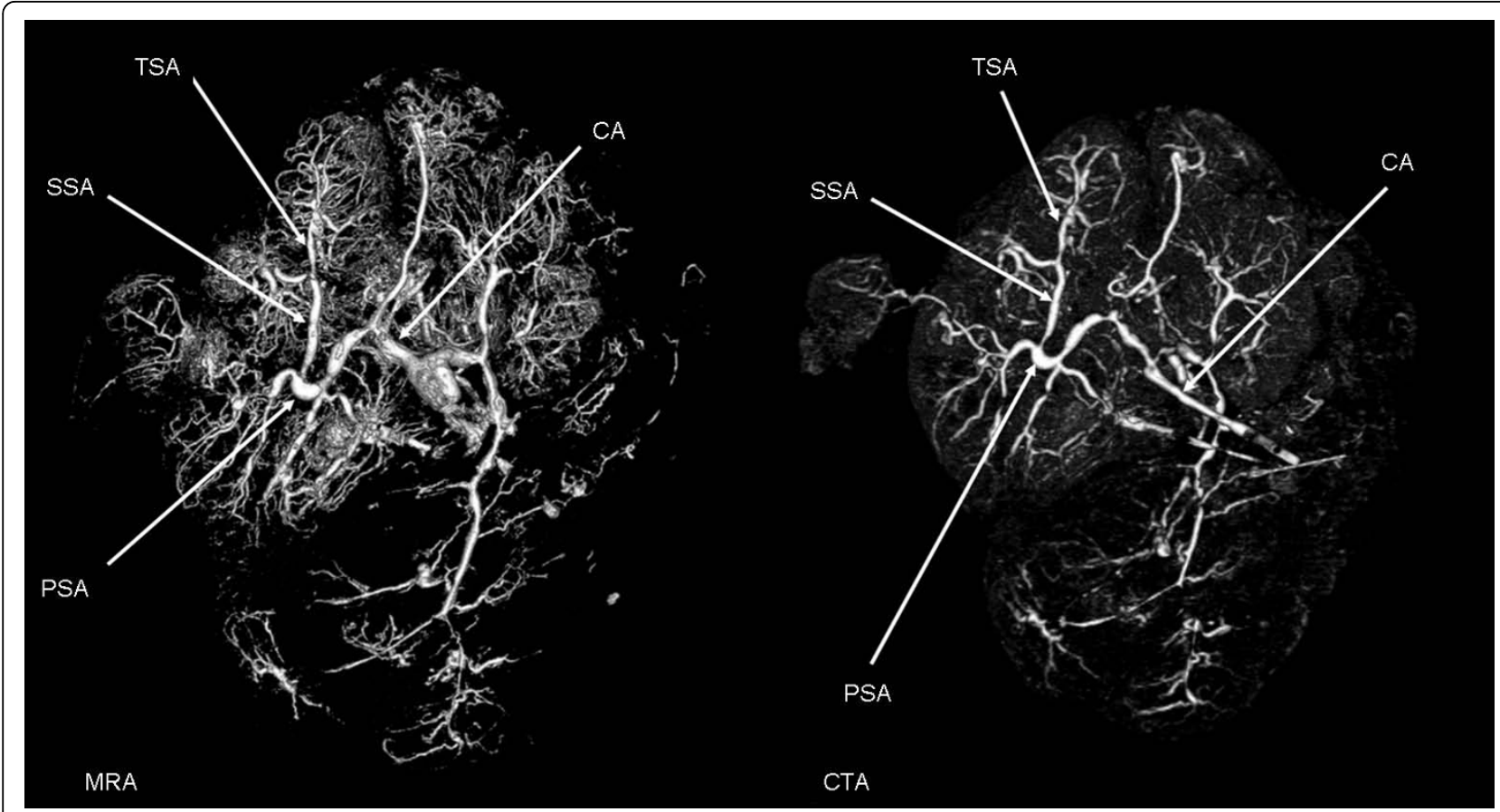

Figure 4 Left: MRA of human placenta; 3D reconstruction made using Osirix software viewed from the foetal side of the placenta. Key: CA, chorionic artery; PSA, primary stem artery; SSA, secondary stem artery; TSA, tertiary stem artery. Right: CTA of human placenta; 3D reconstruction made using Osirix software viewed from the foetal side of the placenta. Key: CA, chorionic artery; PSA, primary stem artery; SSA, secondary stem artery; TSA, tertiary stem artery.

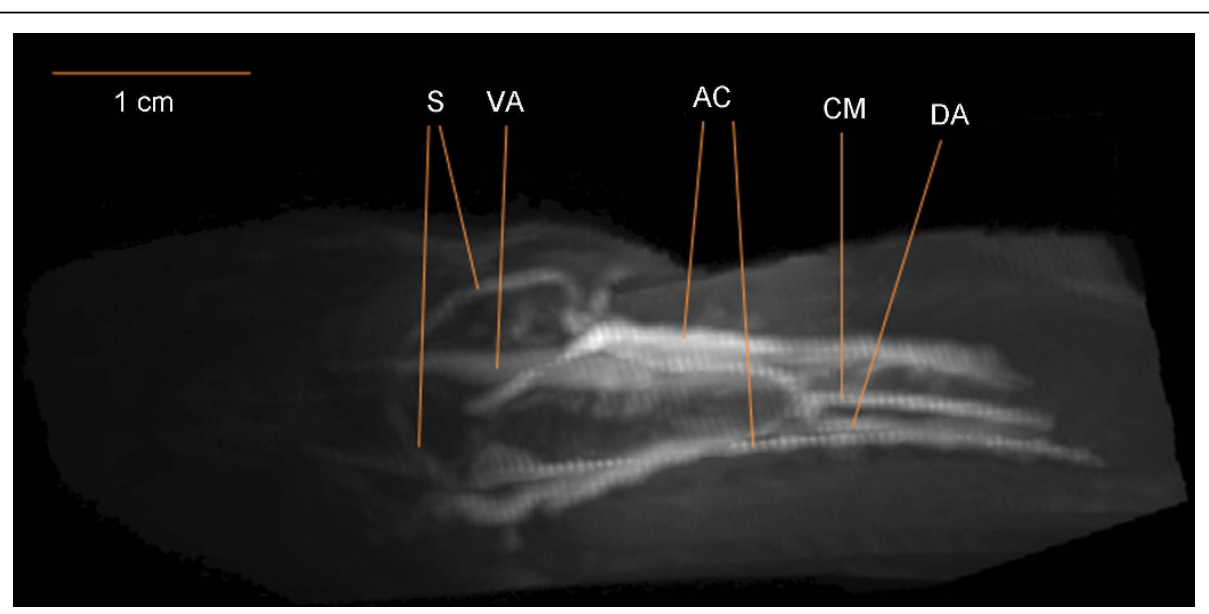

Figure 5 Dorsal view of the region $3-6 \mathrm{~cm}$ caudal to the snout of Monopterus albus perfused with contrast mix 2, with anterior to the left. The image was created by 3D-rendering software following MRI. Key: VA, ventral aorta; DA, dorsal aorta; S, shunt vessels joining ventral and dorsal aorta; CM, celiacomesenteric artery; AC, anterior cardinal veins.

delineate major vessels in the head, such as the ventral and dorsal aortae, the connected shunt vessels and the celiacomesenteric artery. Thanks to the low viscosity of contrast mix 2 , it was possible to delineate the small capillary beds in the head, allowing a 3D representation of the anterior cardinal veins (Figure 6). The advantage of MRA over CTA was its ability to image the vessel structures without including the bone and cartilage, which is of particular importance in this species where the blood vessels and bones lie in close proximity.

\section{Porcine heart}

One female Danish Landrace pig weighing $65 \mathrm{~kg}$ was used for the experiment. Following anaesthesia the pig was moved to the surgical unit where $100 \mathrm{IU} / \mathrm{kg}$ heparin was administered through an ear vein cannula and allowed to 


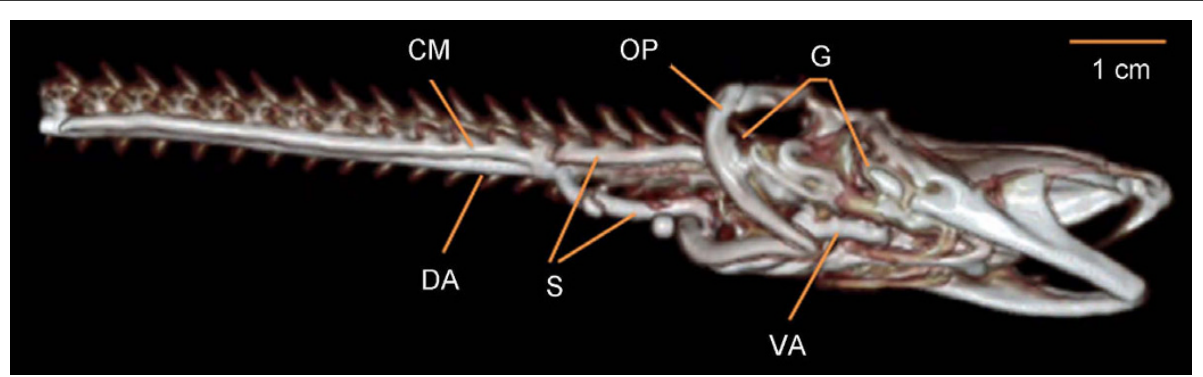

Figure 6 CTA of Monopterus albus using Microfil contrast medium showing a lateral view of the head region; anterior to the right Image created by 3D rendering software following CT. Key: VA, ventral aorta; DA, dorsal aorta; S, shunt vessels joining ventral and dorsal aorta; $\mathrm{CM}$, celiacomesenteric artery; G, right gill region; OP, right operculum.

circulate in the bloodstream for 5 min before euthanasia was induced by an overdose of phenobarbital. Subsequently, thoracotomy was performed and the heart was removed. The proximal part of the left anterior descending artery and right coronary artery were dissected free and a suture was placed around the arteries. A cannula (inner diameter $2.5 \mathrm{~mm}$ ) was introduced into the proximal part of each artery and sutures were tightened around them to prevent retrograde flow of the contrast solution. Next, the coronary arteries were perfused with contrast mix 1, exvivo MRA was performed and the heart was perfused with $2.8 \%$ FineFix prior to CTA (Figure 7). MRA provided detailed information about the blood vessel anatomy and showed the smallest blood vessels in considerably greater number than CTA. Both MRA and CTA showed the right coronary artery (RCA), left anterior descendens (LAD) and the circumflex branch (CX).

\section{Trachemys scripta}

To test whether MRA is feasible for whole animals, two turtles (Trachemys scripta) were perfused with heparinized saline followed by perfusion with contrast mix 1. Perfusion was performed from both arterial and venous sides to make filling of the blood vessels more complete. Next, each animal was cooled with ice to solidify the injected contrast mix. MRA and CTA were performed. Figure 8 shows a 3D reconstruction of the images obtained with MRA and CTA, demonstrating that MRA provided several advantages over CTA. Smaller branches of the blood vessels could be observed by MRA, whereas the turtle shell absorbed the emitted $\mathrm{X}$-ray radiation in CTA, making it difficult to depict blood vessels in close proximity to the shell.

\section{Conclusions}

We have demonstrated a novel experimental method to generate a $3 \mathrm{D}$ representation of blood vessels using MRA specifically applied to ex-vivo studies. We found that MRA provided detailed information similar to or better than conventional CTA. MRA has a great advantage over CTA in its ability to visualize only the vessel configuration without interfering signals from adjacent

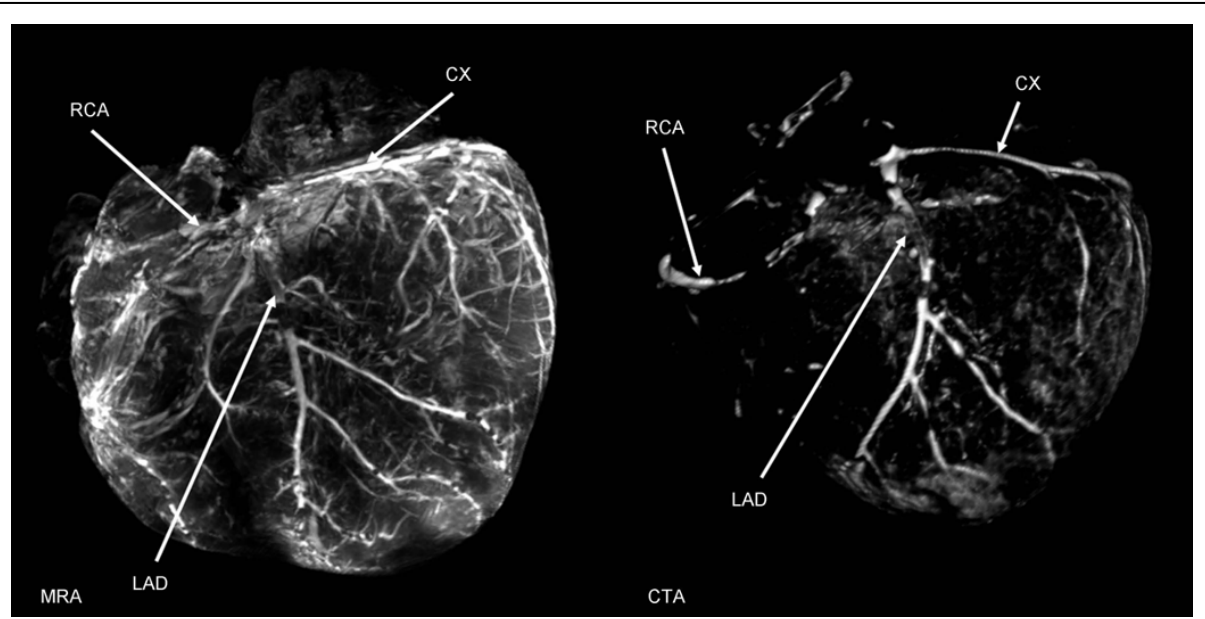

Figure 7 MRA and CTA of the same porcine heart. Left: MRA of heart from female Danish Landrace pig, visualized in a 3D view. Key: LAD, left anterior descending artery; CX, circumflex artery; RCA, right coronary artery. Right: CTA of the heart visualized in a 3D view. Key: LAD, left anterior descending artery; CX, circumflex artery; RCA, right coronary artery. 


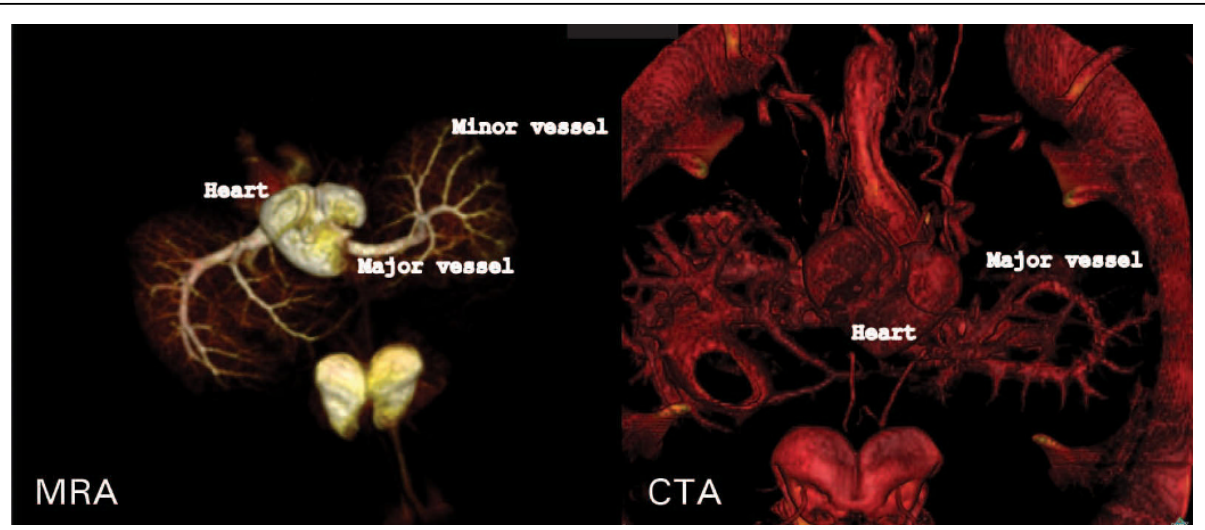

Figure 8 MRA and CTA of a turtle. Left: MRA in a 3D view. Right: CTA in a 3D view.

bones as seen in CTA. In all cases, we found that the best method to obtain high-quality ex vivo MRA was to perfuse the blood vessels with saline containing heparin at $40^{\circ} \mathrm{C}$ immediately after organ harvesting, then lowered into a water-bath at $40^{\circ} \mathrm{C}$ and perfused with contrast solution. In cases where both MRA and CTA must be performed on the same specimen, contrast mix 1 is to be preferred whereas in cases where only MR scans are performed contrast mix 2 is preferable. It was observed that placental vascular tissue becomes leaky when formalin-fixed, leading to increased permeability and outward diffusion of MR contrast agent.

\section{Methods}

\section{Ethics}

This study complied with the Helsinki Declaration. Approval to use human placentas was given by the The Danish National Committee on Biomedical Research Ethics (approval \#M-20080152), and all pregnant woman gave their written consent for the use of their placenta. Approval to include animals in this study was given by the The Danish Ministry of Justice for Animal Experiments Inspectorate (approval \#2008-561-1522).

\section{MRA and CTA}

MRA was performed with clinically available MR systems, a GE Horizon Echospeed LX 1.5 T (GE Healthcare, United Kingdom) and a Philips Achieva $1.5 \mathrm{~T}$ (Philips Medical Systems, Netherlands). Each specimen was positioned in a quadrature radiofrequency head coil. A fast localizer scan was followed by a high-resolution 3D gradient-echo sequence with the following parameters: field-of-view depending on specimen; thickness $1 \mathrm{~mm}$; TR $23.1 \mathrm{~ms}$; TE $1.6 \mathrm{~ms}$ and excitation flip angle $30^{\circ}$. A stack of multiple slices (with no gaps) was acquired, covering the entire specimen of interest with scan durations of 30-60 min. Image resolution was $0.5 \times$ $0.5 \times 0.5 \mathrm{~mm}^{3}$
CTA was performed using a 64-slice Siemens Somatom Definition (Siemens Medical Solutions, Germany) with dual source capacity. Acquisition parameters included a slice collimation of $4 \mathrm{~mm}$; a pitch of $2^{\circ} ; 32$ rotations (resulting in a $25.6 \mathrm{~cm}$ scanning volume) and a matrix size of $512 \times 512$. Acquisition parameters using the Somatom Volume Zoom option included a slice collimation of $4 \times 1 \mathrm{~mm}$, a rotation time of $0.5 \mathrm{~s}, 5-8 \mathrm{~mm}$ table feed/rotation, a matrix size of $512 \times 512$ and scan duration of 25-30 s. Transverse images were reconstructed with a section thickness of $1.25 \mathrm{~mm}$ and were reconstructed at $0.6 \mathrm{~mm}$ intervals.

Data acquired both by MRA and CTA were exported to DICOM format and a 3D reconstruction was performed using either the Mistar http://www.apollomit. com or the Osirix software http://www.osirix-viewer. com, allowing a maximum-intensity-projection in arbitrary directions.

\section{SEM}

Two tissue samples were withdrawn from fresh placental blood vessels and two from formalin-fixated placenta blood vessels. The samples were freeze-dried and mounted on sample holders. The sample holders were placed in an Edward's gold sputter-coater, which covered the sample surface with a thin layer of gold. Subsequently, the samples were placed in a CamScan MaXim 2040 EnVac SEM (CamScan, United Kingdom) and images were obtained using secondary electrons and an Everhart-Thornley detector.

\section{Acknowledgements}

We thank Jesper Thygesen for valuable help during CTA procedures. Troels Thim helped in making the contrast solutions and we thank Jacques Chevalier for SEM assistance.

\section{Author details}

'MR-research Centre, Aarhus University Hospital, Aarhus, Denmark.

${ }^{2}$ Department of Biology, Aarhus University, Aarhus, Denmark. ${ }^{3}$ Department of 


\section{Authors' contributions}

MP constructed the MR sequence appropriate for MRA. HL performed MRA and CTA on the rice field eel. SFP performed porcine surgery. ASR performed MRA on the human placenta and the porcine heart. MP and TW performed MRA on the turtles. LU and LWTB performed CTA on the human placenta, turtle and porcine heart. BGJ, TW, NU, MP, HL, CL and ASR participated in designing the study. MP, HL and ASR participated in drafting the manuscript. All authors read and approved the final manuscript.

Received: 3 July 2009 Accepted: 12 March 2010

Published: 12 March 2010

\section{References}

1. Hoenig MR, Doust JA, Aroney CN, Scott IA: Early invasive versus conservative strategies for unstable angina \& non-ST-elevation myocardial infarction in the stent era. Cochrane Database Syst Rev 2006, 3 : CD004815.

2. Mori S, Kondo C, Suzuki N, Hattori A, Kusakabe M, Endo M: Volumetric coronary angiography using the 256-detector row computed tomography scanner: comparison in vivo and in vitro with porcine models. Acta Radiol 2006, 47:186-191.

3. Jin H, Zeng MS, Ge MY, Yang S, Chen CZ, Shen JZ, Li RC: A study of in vitro and in vivo MR of free-breathing whole-heart 3D coronary angiography using parallel imaging. Int I Cardiovasc Imaging 2009, 25(Suppl 1):121-129.

4. Lim HB, Hur G, Kim SY, Kim YH, Kwon SU, Lee WR, Cha SJ: Coronary stent fracture: detection with 64-section multidetector $\mathrm{CT}$ angiography in patients and in vitro. Radiology 2008, 249:810-819.

5. Wintermark M, Jawadi SS, Rapp JH, Tihan T, Tong E, Glidden DV, Abedin S, Schaeffer S, Acevedo-Bolton G, Boudignon B, Orwoll B, Pan X, Saloner D: High-resolution CT imaging of carotid artery atherosclerotic plaques. Am J Neuroradiol 2008, 29:875-882.

6. Chade AR, Zhu X, Lavi R, Krier JD, Pislaru S, Simari RD, Napoli C, Lerman A, Lerman LO: Endothelial progenitor cells restore renal function in chronic experimental renovascular disease. Circulation 2009, 119:547-557.

7. Kominato $Y$, Fujikura T, Hata $Y$, Matsui K, Takizawa H: A case of postoperative hemorrhage after a hysterectomy in which a bleeding point of the left uterine artery was identified by postmortem angiography. Leg Med (Tokyo) 2004, 6:187-189.

8. Jackowski C, Persson A, Thali MJ: Whole body postmortem angiography with a high viscosity contrast agent solution using poly ethylene glycol as contrast agent dissolver. J Forensic Sci 2008, 53:465-468.

9. Wischgoll T, Choy JS, Ritman EL, Kassab GS: Validation of image-based method for extraction of coronary morphometry. Ann Biomed Eng 2008, 36:356-368.

10. Zhou YQ, Davidson L, Henkelman RM, Nieman BJ, Foster FS, Yu LX, Chen XJ: Ultrasound-guided left-ventricular catheterization: a novel method of whole mouse perfusion for microimaging. Lab Invest 2004 84:385-389.

11. Gordon Z, Elad D, Almog R, Hazan Y, Jaffa AJ, Eytan O: Anthropometry of fetal vasculature in the chorionic plate. J Anat 2007, 211:698-706.

12. Jauniaux E, Moscoso JG, Vanesse M, Campbell S, Driver M: Perfusion fixation for placental morphologic investigation. Hum Pathol 1991, 22:442-449.

13. Munshi JSD, Ghosh TK, Ojha J, Olson KR: Vasculature of the head and respiratory organs in an obligate air-breathing fish, the swamp eel Monopterus (= Amphipnous) cuchia. J Morphol 1990, 203:181-201.

doi:10.1186/1472-6793-10-3

Cite this article as: Rasmussen et al:: High-resolution ex vivo magnetic resonance angiography: a feasibility study on biological and medical tissues. BMC Physiology 2010 10:3.

\section{Submit your next manuscript to BioMed Central and take full advantage of:}

- Convenient online submission

- Thorough peer review

- No space constraints or color figure charges

- Immediate publication on acceptance

- Inclusion in PubMed, CAS, Scopus and Google Scholar

- Research which is freely available for redistribution

Submit your manuscript at www.biomedcentral.com/submit
Biomed Central 